\title{
Dobutamine magnetic resonance imaging as a predictor of myocardial functional recovery after revascularisation
}

\author{
R J Trent, G D Waiter, G S Hillis, F I McKiddie, T W Redpath, S Walton
}

\begin{abstract}
Objective-To assess the use of dobutamine magnetic resonance imaging (MRI) as a preoperative predictor of myocardial functional recovery after revascularisation, comparing wall motion and radial wall thickening analyses by observer and semi-automated edge detection.

Patients-25 men with multivessel coronary disease and resting wall motion abnormalities were studied with preoperative rest and stress MRI.

Main outcome measures-Observer analysis for radial wall thickening was compared with a normal range, while wall motion analysis used a standard four point scale. Semi-automated analysis was performed using an edge detection algorithm. Segments displaying either improved or worsened thickening or motion with dobutamine were considered viable. Postoperative rest images were performed 3-6 months after coronary artery bypass grafting (CABG) for comparison.

Results-For observer analysis the values for sensitivity and specificity were $50 \%$ and $72 \%$ for wall motion, with respective values of $50 \%$ and $68 \%$ for thickening. With semi-automated edge detection the figures for motion were $60 \%$ and $73 \%$, with corresponding values of $79 \%$ and $58 \%$ for thickening. Combining thickening and motion for the semi-automated method to describe any change in segmental function yielded a sensitivity of $71 \%$ and specificity of $70 \%$.

Conclusions-Dobutamine MRI is a reasonably good predictor of myocardial functional recovery after CABG. The use of semi-automated edge detection analysis improved results. (Heart 2000;83:40-46)
\end{abstract}

Keywords: dobutamine, magnetic resonance imaging myocardial viability; coronary artery bypass grafting

At present, the finding of severe left ventricular dysfunction is still considered, in many cases, to be a bar to successful coronary revascularisation, being associated with a significantly higher perioperative mortality and morbidity. ${ }^{1}$ The occurrence of spontaneous contractile improvement after coronary artery bypass grafting (CABG) in chronic, severe, left ventricular dysfunction has been well described, however, and attributed to the presence of "hibernating" myocardium. ${ }^{2}$ The clinical diagnosis of hibernation is essentially retrospective, with the need for reliable preoperative diagnosis of hibernation therefore of cardinal importance. While the presence of a flow/metabolic mismatch at positron emission tomography (PET) scanning remains the gold standard for preoperative diagnosis, this investigation is not available outside a few centres in the UK and Europe. $\mathrm{Tl}^{201}$ reinjection scintigraphy, ${ }^{3-7} \quad \mathrm{Tc}^{99 \mathrm{~m}}$ sestamibi scintigraphy, ${ }^{4-10}$ and low dose dobutamine echocardiography ${ }^{4} 7^{11-13}$ have been investigated extensively.

More recently, the use of dobutamine magnetic resonance imaging (MRI) has also been described. ${ }^{14-20}$ The majority of echocardiographic and MRI series have used observer wall motion analysis to quantify the response to dobutamine stimulation and subsequent postoperative recovery. Assessment of systolic wall thickening, rather than endocardial motion, negates the effect of paradoxical septal motion associated with median sternotomy, ${ }^{21}$ and is less susceptible to the effects of cardiac translation and tethering. ${ }^{10}$ Baer and colleagues describe impressive results using dobutamine MRI ${ }^{18-20}$ although other groups have failed to reproduce this. ${ }^{17}$

In this study we attempt to assess the diagnostic performance of MRI, using both manual and semi-automated analysis to determine changes in radial wall thickening and radial wall motion. Postoperative rest MRI was used to determine improvement in segmental left ventricular function.

\section{Methods}

PATIENTS

Forty men with previous myocardial infarction awaiting CABG for conventional reasons (symptoms unresponsive to medical treatment, left main stem disease, or triple vessel disease with left ventricular impairment) were studied. All had multivessel coronary disease and at least one major regional wall motion abnormality at ventriculography. The mean (SD) left ventricular ejection fraction (LVEF) was 54 (14.5)\%. Mean age was 60 years. Medications were withheld on the morning of study. Patient details are given in table 1. Of the initial subjects, three were excluded because of recent unstable symptoms and three produced images of insufficient quality. One study was abandoned because of technical reasons and one was discontinued because of tachyarrhythmia. Thus 32 patients completed preoperative imaging. Postoperative imaging was performed
Accepted for publication 30 July 1999 
Table 1 Patient characteristics

\begin{tabular}{|c|c|c|c|c|}
\hline Patient & Age (years) & Sex & Infarct site & LVEF \% (pre/post) \\
\hline 1 & 65 & M & Anterior & $42 / 45$ \\
\hline 2 & 60 & $M$ & Anterior/inferior & $38 / 19$ \\
\hline 3 & 51 & $M$ & Inferior & $66 / 65$ \\
\hline 4 & 66 & $M$ & Inferior & $63 / 65$ \\
\hline 5 & 47 & $M$ & Inferior & $39 / 43$ \\
\hline 6 & 71 & $M$ & Anterior & $43 / 26$ \\
\hline 7 & 61 & $M$ & Anterior & $52 / 38$ \\
\hline 8 & 69 & $M$ & Anterior & $47 / 34$ \\
\hline 9 & 78 & M & Inferior & $47 / 35$ \\
\hline 10 & 71 & $M$ & Anterior/inferior & $68 / 64$ \\
\hline 11 & 68 & M & Inferior & $56 / 44$ \\
\hline 12 & 63 & $M$ & Anterior & $57 / 46$ \\
\hline 13 & 56 & $M$ & Inferior & $50 / 55$ \\
\hline 14 & 41 & M & Inferior & $48 / 39$ \\
\hline 15 & 67 & $M$ & Inferior & $84 / 73$ \\
\hline 16 & 60 & $M$ & Inferior & $47 / 33$ \\
\hline 17 & 67 & $M$ & Inferior & $46 / 55$ \\
\hline 18 & 67 & $M$ & Anterior & $65 / 70$ \\
\hline 19 & 72 & M & Inferior & $75 / 64$ \\
\hline 20 & 66 & $M$ & Anterior & $54 / 60$ \\
\hline 21 & 67 & $M$ & Inferior & $32 / 46$ \\
\hline 22 & 66 & $M$ & Inferior & $17 / 19$ \\
\hline 23 & 56 & $M$ & Anterior & $29 / 41$ \\
\hline 24 & 79 & $M$ & Anterior & $20 / 34$ \\
\hline 25 & 64 & $M$ & Inferior & $67 / 63$ \\
\hline Mean (SD) & $64(8.7)$ & & & $52.7(16) / 47.4(17)$ \\
\hline
\end{tabular}

3-6 months after revascularisation. Three patients declined to return for postoperative imaging and a further four produced images of insufficient quality for data analysis. Thus, a total of 25 pre- and postoperative paired MRI scans were available for analysis. These formed the basis of this study.

IMAGING

Patients were imaged using a 0.95 Tesla Siemens (Erlangen, Germany) Impact MRI scanner with standard body transmit and receive radiofrequency coil. Rapid gradient echo "Turbo-FLASH" left ventricular localisation was performed with the coronal plane defining the long axis. Multiple temporal cine-MR images were obtained in the short axis plane using a fast imaging with steady state precision (FISP) pulse sequence with gradient motion rephasing, employing a flip angle of $30^{\circ}$, an echo time of $12 \mathrm{~ms}$, and a frame separation of $50 \mathrm{~ms}$. Image acquisitions were ECG gated with an $\mathrm{R}$ wave trigger, producing a maximum of 12 frames per cycle. The field of view used was $400 \mathrm{~mm}$, with an image thickness of $10 \mathrm{~mm}$ and three averages obtained. The acquisition matrix was reconstructed using the standard two dimensional Fourier transformation algorithm and interpolated to $256 \times 256$ for data storage and display purposes. Four non-contiguous slices were

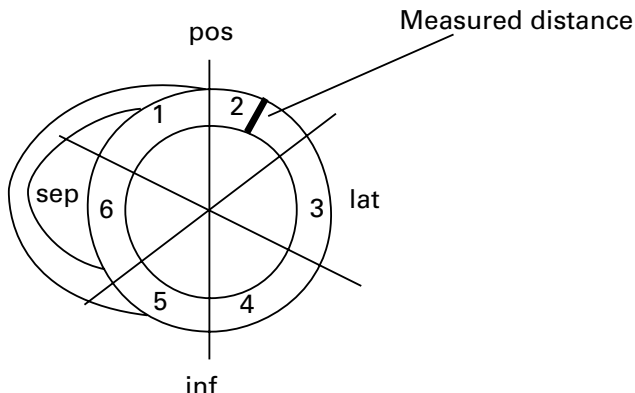

Figure 1 Schematic of short axis segmental views. pos, posterior; inf, inferior; lat, lateral; sep, septal. obtained from base to left ventricular apex. Preoperative rest and stress images were performed sequentially, using a dobutamine infusion titrated (mean (SD) dose $15(4.9) \mu \mathrm{g} /$ $\mathrm{kg} / \mathrm{min}$ ) to induce a $50 \%$ increase in mean basal heart rate from 65 (13) beats per minute to 95 (11) beats per minute. The mean duration of in-scanner time was approximately 30 minutes.

ANALYSIS

Images were studied by three independent observers: a cardiologist (observer 3 ) and two medical physicists from the MRI department. Dysfunctional segments were identified for each subject from the preoperative scans and these segments were then characterised as viable/non-viable on the basis of the response to dobutamine. These data were then tested against the postrevascularisation results; interpretation of postoperative resting MR images was performed blinded to the results of the dobutamine study and in a random manner. Calculation of sensitivity and specificity was performed from standard formulae. This was done for all abnormal segments, and also broken down into akinetic (score 1) and hypokinetic (score 2) segments, as there is some evidence to suggest that the predictive power of dobutamine (echo) is greatest in the most severely dyssynergic segments. ${ }^{22-24}$

MRI data were analysed from four short axis views divided into a six segment "cartwheel" (fig 1). Left ventricular wall thickness was measured in the centre of each segment, perpendicular to the epicardium. Manual measurement of radial wall thickening was made with electronic calipers and compared with the existing normal range for this centre $(n=31$, table 2 ), a reduction of $>1$ SD below normal being considered as reduced. A value of $1 \mathrm{SD}$ was used because of the large variation in the normal database. Semisubjective regional wall motion observer analysis was scored on a scale of $0-3 \quad(0=$ dyskinesis; $1=$ akinesis; $2=$ hypokinesis; $3=$ normal $)$ and a reduced regional wall motion as a score of $\leqslant 2$. Global left ventricular function was not formally assessed as a criterion of study entry, but was measured incidentally as the LVEF pre- and postoperatively by MRI. ${ }^{25} 26$

Data were also analysed by semi-automated edge detection. ${ }^{27}$ After identification of the left ventricular centre point, a radial search at $2^{\circ}$ intervals determined the point of maximum pixel intensity gradient, taken as the radial distance of the border from the centre point. Data were then converted into $\mathrm{x}$ and $\mathrm{y}$ coordinates for epicardial/endocardial borders, and fitted to a two dimensional polynomial function (degree $=6$ ), allowing interpolation of missing or misrepresented regions. Wall thickness was given by the distance between the interpolated borders at points corresponding to those used in the manual method. Semi-automated wall thickening measurements were made in the centre of the segment to coincide as closely as possible with the manual measurement, therefore no averaging was done. There was no difference in the normal range for segmental 
Table 2 Normal range for manual wall thickening determination (cm) given as mean $(S D)$

\begin{tabular}{lllllll}
\hline & \multicolumn{2}{l}{ Segment } & \multicolumn{1}{l}{} & \\
\cline { 2 - 6 } Slice & 1 & 2 & 3 & 4 & 5 & 6 \\
\hline 1 & $0.61(0.35)$ & $0.66(0.38)$ & $0.57(0.35)$ & $0.53(0.45)$ & $0.32(0.3)$ & $0.43(0.24)$ \\
2 & $0.42(0.31)$ & $0.78(0.24)$ & $0.94(0.46)$ & $1.01(0.41)$ & $0.6(0.32)$ & $0.36(0.25)$ \\
3 & $0.74(0.28)$ & $0.89(0.34)$ & $1.17(0.4)$ & $1.04(0.35)$ & $0.63(0.37)$ & $0.51(0.29)$ \\
4 & $0.72(0.25)$ & $0.88(0.28)$ & $0.89(0.33)$ & $0.69(0.42)$ & $0.65(0.34)$ & $0.57(0.34)$ \\
\hline
\end{tabular}

thickening determined by the semi-automated method ( $\mathrm{p}=\mathrm{NS}$, paired $t$ test). Endocardial wall motion was defined as the percentage change in the distance from the endocardium to the centre of the left ventricle from end diastole to end systole.

The relation between segmental response to dobutamine and global functional improvement within individuals postoperatively was not assessed during the study period, though retrospective analysis was performed. The mean number of dobutamine viable segments by semi-automated edge detection of systolic wall thickening per patient was related to the change in LVEF postoperatively by linear regression. Also, comparison of the mean number of segments in those subjects with improved LVEF was compared with those subjects without improvement.

STATISTICS

A segment was deemed to have abnormal wall thickening if its value was found to be more than $1 \mathrm{SD}$ below the mean value. Sensitivity and specificity of dobutamine MRI was determined using postoperative MRI scans as the gold standard for segmental recovery. Sensitivity, specificity, and diagnostic accuracy were calculated using standard formulas and confidence intervals (CIs) determined at the 95\% level..$^{28}$ Inter- and intraobserver agreement was determined by calculating the mean (SD) of the difference between wall thickness measurements. $\kappa$ statistics were determined for the difference in observer scoring of wall motion. All other results are presented as mean (SD) (table 3). Comparisons of global left ventricular function (LVEF) between groups were made by paired $t$ test (significance level at $\mathrm{p} \leqslant 0.05$ ) and by linear regression.

\section{Results}

The ranges (with 95\% CIs) of values obtained for sensitivity and specificity for each individual observer are given in table 3. For further discussion the results of wall motion and thickening analyses for observer 3 are quoted. The results for semi-automated edge detection analysis are given for observer 1 only.

Table 3 Sensitivity of dobutamine stress MRI for the detection of hibernating by semisubjective observer analysis

\begin{tabular}{|c|c|c|c|c|}
\hline \multirow[b]{2}{*}{ Observer } & \multicolumn{2}{|l|}{ Motion } & \multicolumn{2}{|l|}{ Thickening } \\
\hline & $\begin{array}{l}\text { Sensitivity } \\
(\%)(95 \% C I)\end{array}$ & $\begin{array}{l}\text { Specificity } \\
(\%)(95 \% \text { CI) }\end{array}$ & $\begin{array}{l}\text { Sensitivity } \\
(\%)(95 \% C I)\end{array}$ & $\begin{array}{l}\text { Specificity } \\
(\%)(95 \% C I)\end{array}$ \\
\hline 1 & $52(43$ to 61$)$ & 65 (57 to 73$)$ & $70(63$ to 77$)$ & 60 (52 to 68$)$ \\
\hline 2 & $40(35$ to 45$)$ & 85 (79 to 91$)$ & $52(44$ to 60$)$ & 66 (60 to 72$)$ \\
\hline 3 & $50(44$ to 56$)$ & $72(67$ to 77$)$ & $50(44$ to 56$)$ & 68 (62 to 74$)$ \\
\hline Mean & $48(42$ to 54$)$ & $80(76$ to 84$)$ & $55(49$ to 61$)$ & $65(60$ to 70$)$ \\
\hline Semi-automated edge detection & $60(55$ to 70$)$ & $73(70$ to 76$)$ & $79(75$ to 83$)$ & 58 (54 to 62$)$ \\
\hline
\end{tabular}

WALL MOTION

A total of $588 / 600$ segments (98\%) were suitable for wall motion analysis. Of these 278 $(47 \%)$ had a score of 2 at rest, with $111(40 \%)$ having an improved score with dobutamine stimulation (observer 3). Improved wall motion was seen in 149 (54\%) postoperatively. The concordance between a positive test result and subsequent postoperative improvement was poor, however, with a sensitivity and specificity of 50\% (75/149) and 72\% (93/129), respectively. A total of $131 / 588$ (22\%) segments were akinetic or dyskinetic (wall motion score $\leqslant 1)$ at rest. Of these, $61(47 \%)$ showed an improved score with dobutamine stimulation, and $58(44 \%)$ a postoperative improvement. The sensitivity and specificity was $60 \%$ (35/58) and $64 \%$ (47/73), respectively. A further 42 segments with a resting wall motion score of $\leqslant 2$ showed a worsening score with dobutamine. Of these, nine segments showed postoperative improvement. Inclusion of these data in the analysis improved the sensitivity obtained $(61 \%)$ at the expense of worsened specificity $(58 \%)$.

\section{WALL THICKENING}

A total of $511 / 600$ segments (85\%) were suitable for measurement of regional wall thickening. Of the 511 segments, 267 (52\%) showed reduced wall thickening at rest. Of these, $107(40 \%)$ showed improvement with dobutamine stimulation and 118/267 (44\%) segments improved postoperatively. The concordance between improved wall thickening and postoperative improvement was, however, once again poor, with a sensitivity of $50 \%$ (59/ $118)$ and a specificity of $68 \%(101 / 149)$. No data for ischaemic segments were available for wall thickening, as the normal range for wall thickening could not be subdivided into a greater degree of abnormality than $>1$ SD below normal.

\section{SEMI-AUTOMATED EDGE DETECTION}

Out of 516 segments ( $86 \%$ ) suitable for semiautomated edge detection analysis, $227(43 \%)$ had reduced wall motion at rest, with $66(29 \%)$ having an improved score during dobutamine stimulation. Improved wall motion was seen in $48(21 \%)$ postoperatively. The concordance between a positive test result and subsequent postoperative improvement was poor, however, with a predictive sensitivity and specificity of $40 \%(19 / 48)$ and $74 \%$ (132/179), respectively. Combination of wall thickening and wall motion data to show any improvement in segmental function ${ }^{5}$ revealed a sensitivity of $68 / 114(60 \%)$ and a specificity of $163 / 232$ $(70 \%)$.

Of the 516 segments suitable for semiautomated edge detection analysis, 119/516 $(23 \%)$ segments showed reduced wall thickening at rest. Of these, $71(60 \%)$ showed improvement with dobutamine stimulation and 66/119 (55\%) segments improved postoperatively. The concordance between improved wall thickening with dobutamine and postoperative improvement was, however, once again 


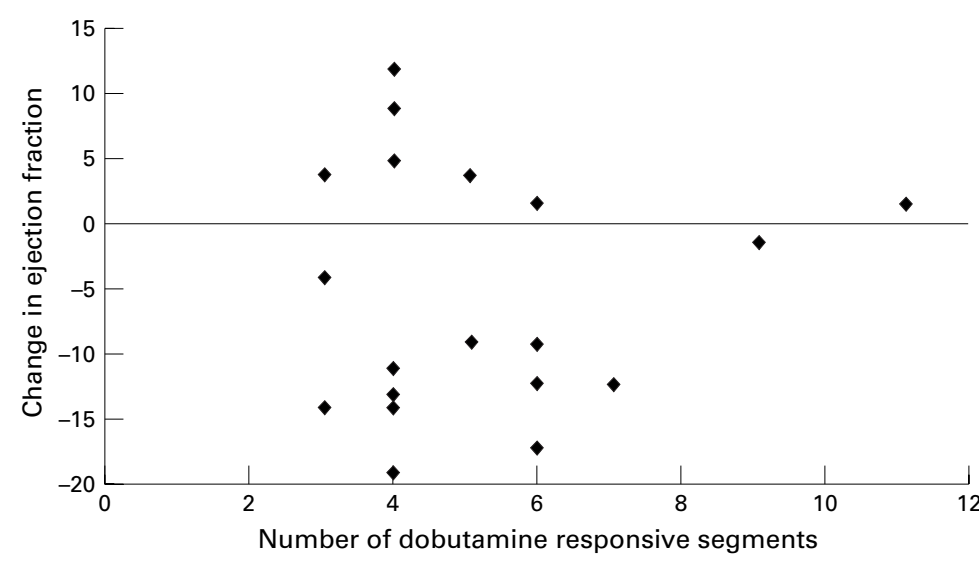

Figure 2 Linear regression of segmental response to dobutamine versus change in left ventricular ejection fraction within individuals.

poor, with a sensitivity of $74 \%(49 / 66)$ and a specificity of $58 \%(31 / 53)$.

Inclusion of segments with an ischaemic response (worsened wall motion) to dobutamine stimulation produced an improved sensitivity for wall motion of $60 \%$ (29/48), with no change in specificity of $73 \%(132 / 179)$, and corresponding values of $79 \%(52 / 66)$ and $58 \%$ $(31 / 53)$ for wall thickening. Data for wall thickening were produced from a count of those segments with resting wall motion abnormality ( $\leqslant 1$ SD below normal) which became more abnormal ( $\leqslant 2 \mathrm{SD}$ ) during dobutamine stimulation. Combination of wall thickening and wall motion data as above, to determine any change in segmental function with dobutamine stimulation, revealed a sensitivity of $71 \%(81 / 114)$ and a specificity of $70 \%$ (163/232).

GLOBAL LEFT VENTRICULAR FUNCTION

There was a trend towards a reduction in mean LVEF postoperatively, with a fall from $54 \%$ to $46 \%$, though this did not reach significance. There was no demonstrable relation between the number of dobutamine viable segments preoperatively and change in LVEF $(r=0.03)$ between pre- and postoperative scans (fig 2). Nine out of 25 subjects showed an increased LVEF postrevascularisation. However, analysis of the mean number of dobutamine viable segments preoperatively was not significantly different between these subjects and those with no increase, or decreased ejection fraction $(p=0.40)$. There was a trend towards a lower preoperative LVEF in those patients showing postoperative improvement $(41.8 \% v 54.7 \%$ in non-improvers), though this did not reach significance $(\mathrm{p}=0.14)$.

OBSERVER VARIABILITY

All images from the 25 patients studied were assessed by three independent observers to allow calculation of interobserver variability. The intraobserver variability was determined by repeated measurement from six of the preoperative images performed by one observer. Radial wall thickness measurements are given as the mean (SD) difference between measurements. The difference in wall motion
Table 4 Interobserver and intraobserver variation

\begin{tabular}{llllll}
\hline Comparison & $\begin{array}{l}\text { Mean } \\
\text { difference }(\mathrm{cm})\end{array}$ & $\begin{array}{l}S D \\
(\mathrm{~cm})\end{array}$ & $\begin{array}{l}\text { Segments } \\
(\mathrm{n})\end{array}$ & $\kappa$ & $\begin{array}{l}\text { Segments } \\
\text { for } \kappa(n)\end{array}$ \\
\hline $1-2$ & 0.06 & 0.43 & 3564 & 0.293 & 1788 \\
$1-3$ & -0.07 & 0.50 & 3564 & 0.114 & 1788 \\
$2-3$ & 0.13 & 0.48 & 3564 & 0.229 & 1788 \\
$1-1$ & -0.003 & 0.38 & 144 & & \\
\hline
\end{tabular}

Observer 1, GDW; observer 2, TWR; observer 3, RJT.

scores is given by the $\kappa$ statistic. The results of these comparisons are shown in table 4 .

\section{Discussion}

Reliable preoperative identification of viable myocardium remains the ultimate aim in the management of the patient with severe left ventricular dysfunction and coronary artery disease. Survival can be improved by revascularisation, ${ }^{29-31}$ although the mortality and morbidity of CABG is increased in this group. ${ }^{1}$ Notwithstanding the lack of randomised controlled trials, Eitzman and colleagues have shown a mortality benefit from CABG in patients with severe left ventricular dysfunction and multivessel coronary disease in the presence of flow metabolism mismatch at PET. ${ }^{31}$ Likewise, Di Carli and colleagues have suggested that prognosis is worse in patients with unrevascularised, PET viable myocardium than in those undergoing revascularisation, or having completed infarction. ${ }^{32}$

The uptake of PET imaging as an investigative tool for clinical, rather than research, use is limited by a lack of availability outside a few centres in Europe and the USA, and other methods are likely to be required for this reason. In a meta-analysis of currently available techniques for prediction of functional recovery after revascularisation in patients with left ventricular dysfunction caused by chronic coronary artery disease, Bax and colleagues describe values for sensitivity of $84 \%$ and specificity of $81 \%$ for low dose $(5-10 \mu \mathrm{g} / \mathrm{kg} / \mathrm{min})$ dobutamine echocardiography from 16 studies. ${ }^{33}$

MRI offers theoretical advantages over echocardiography in the assessment of myocardial viability. Chiefly, the use of objectively defined systolic wall thickening as a measure of viability may be superior to semisubjective observer wall motion analysis. Additionally, wall motion analysis is vulnerable to artefacts resulting from paradoxical septal motion, cardiac translation, and tethering. ${ }^{11}{ }^{21}$ Baer and colleagues have recently described a sensitivity of $89 \%$ and specificity of $94 \%$ for systolic wall thickening with low dose dobutamine MRI. ${ }^{20}$ Gunning and colleagues, however, describe values much closer to our own, with sensitivity and specificity of $50 \%$ and $81 \%$ for wall motion, and $45 \%$ and $83 \%$ for wall thickening, respectively, again using a low dose regimen. ${ }^{17}$

Although the majority of echocardiography based and MRI work has described the use of low dose dobutamine for viability study, a number of studies have suggested the use of higher dobutamine doses may be required to optimise the diagnostic yield. Sawada and colleagues used low $(5-10 \mu \mathrm{g} / \mathrm{kg} / \mathrm{min})$ and high dose (30 (10) $\mu \mathrm{g} / \mathrm{kg} / \mathrm{min})$ regimens. ${ }^{34}$ Of those dyssynergic myocardial segments shown to be 
viable at PET, wall motion improved with low dose dobutamine in $70 \%$, though in $30 \%$ only at high dose. Sklenar and colleagues ${ }^{35}$ found, in experimental work, that a dose of $15 \mu \mathrm{g} / \mathrm{kg} / \mathrm{min}$ was required to produce maximal wall thickening after myocardial infarction, while Afridi and colleagues ${ }^{13}$ found that administration of both low and high dobutamine doses was necessary to optimise the predictive power of dobutamine echocardiography. Moreover, in the reworsening phase of those segments showing a biphasic response, this was usually seen with doses of $\geqslant 20 \mu \mathrm{g} / \mathrm{kg} / \mathrm{min}$. Similarly, Cornel and colleagues used a combined low $(5-10 \mu \mathrm{g} / \mathrm{kg} /$ $\mathrm{min})$ and high dose ( $\leqslant 40 \mu \mathrm{g} / \mathrm{kg} / \mathrm{min})$ regimen, showing that a biphasic response was the only independent predictor of an improved LVEF postrevascularisation. ${ }^{24}$

An intermediate dobutamine dose (mean $15 \mu \mathrm{g} / \mathrm{kg} / \mathrm{min}$ ) was used in this study, in order to minimise potential underdiagnosis of viability reported with standard low dose regimens ${ }^{13} 24345$ and reduce the in-scanner time associated with multiple dose imaging. ${ }^{36}$ The development of worsening wall motion abnormality in approximately $10 \%$ of already abnormal segments suggests ischaemia, and therefore viability, in these segments. ${ }^{37}$ Indeed, the inclusion of segments with worsened wall motion or thickening abnormality post dobutamine improved the sensitivity of MRI in this series, though only in association with the use of semi-automated edge detection analysis. However, a further $8 \%$ of abnormal segments at rest MRI, as judged by wall motion, and $12 \%$ by wall thickening showed no improvement during dobutamine stimulation, although postoperative improvement was seen. It is possible that these areas may have represented a biphasic response, where the improvement in wall motion occurring at a lower level of dobutamine stimulation is followed by "reworsening" at higher dose levels, and is therefore not apparent with imaging at a single dose. This phenomenon was not well described at the time of drawing up our study protocol, though with retrospect the lack of multiple dose level imaging is a major limitation.

Earlier work by Cornell and colleagues ${ }^{23} 24$ found that the specificity of low dose dobutamine echocardiography was reduced in mildly dysfunctional segments. Kaul and colleagues have shown similar findings. ${ }^{22}$ Our data for observer wall motion analysis are not entirely consistent with these findings, showing a slightly better sensitivity and slightly lower specificity in segments with greater resting wall motion abnormality, with a sensitivity of $50 \%$ and specificity of $72 \%$ for all abnormal segments, and respective values of $60 \%$ and $64 \%$ in akinetic or dyskinetic segments. The reason for this is not clear, though the relatively small sample size and difference between the study populations may be contributory factors. Given these differences, and the variation in dobutamine stress protocols, it is hard to draw any further conclusions from this aspect of the study results.

Further explanation of the discrepancy between our results and those of Baer and colleagues $^{18-20}$ may be found in analysis of the respective study populations. It is known that the contractile response to dobutamine stimulation of hibernating myocardium, as defined by flow-metabolic mismatch with PET, is poor, in contrast to stunned myocardium. ${ }^{38-40}$ The majority of subjects in this series had been referred for revascularisation for conventional reasons of symptomatic relief, with or without prognostic implications. Regional wall motion abnormality in this situation may well represent repeated episodes of myocardial stunning, rather than hibernation. ${ }^{34} 40$

In a study of patients with ischaemic left ventricular dysfunction Sawada found the majority of PET viable segments with resting wall motion abnormality had normal perfusion and metabolism, suggesting that stunning, rather than hibernation per se, was the cause of the dysfunction. ${ }^{34}$ In addition, improvement in wall motion was approximately twice as likely in those regions with normal perfusion and metabolism (that is, stunned) than in those with reduced tracer uptake or mismatch (that is, hibernating). A biphasic response identified segments with normal perfusion and metabolism in nearly $90 \%$ of cases, these being subtended by vessels with $70 \%$ stenosis. It is important to note, moreover, that the prevalence of severe global left ventricular dysfunction in this series was low, with only $15 \%$ of patients studied having a resting LVEF of $<40 \%$, as determined by MRI at the time of study, with a mean LVEF of 54 (14.5)\%. The mean normal value for MRI determined LVEF in our centre is $65(12.5) \%$. In contrast, Baer and colleagues studied patients referred to a tertriary centre with significantly greater left ventricular dysfunction (mean LVEF $42 \%$ ). ${ }^{20}$ By Bayesian theory, therefore, the yield obtained in this series is likely to be lower.

Perhaps surprisingly, we found no association between the amount of dobutamine viable myocardium (mean segmental response) and change in LVEF postrevascularisation. This is at variance with Baer and colleagues ${ }^{20}$ and again seems most likely to be a manifestation of the comparatively better preoperative left ventricular function in our study population. Interestingly, there was a discernable trend towards lower preoperative LVEF in those patients showing improved postoperative LVEF. These findings are consistent with the dictum that the diagnosis of myocardial viability is of greatest importance in those with significantly impaired left ventricular function.

The results of this series are remarkably similar to those obtained by Gunning and colleagues ${ }^{17}$ using a low dose dobutamine protocol, without breath hold imaging and a larger (nine segment) segmental imaging method. Both of these studies show a modest sensitivity, with good specificity for the preoperative prediction of functional recovery after revascularisation in dysfunctional myocardial segments. The results obtained by Baer and colleagues are considerably more impressive, with sensitivity and specificity of around $90 \% .{ }^{20}$ Although our dobutamine protocol differed from that of Baer and colleagues, the low dose 
protocol was common to both the Gunning and Baer studies. ${ }^{17}{ }^{20}$ The technique of rapid breath hold imaging was unique to Baer's series, not being available at the outset of our study. Moreover, they excluded myocardial segments not subtended by a $70 \%$ coronary stenosis unlike ourselves, and used high dose nitrates periprocedurally to maximise coronary vasodilation. ${ }^{41} 42$

Further limitations of this study include the possibility that the time to recovery of dyssynergic myocardium after CABG in our subjects may have exceeded the 3-6 month period in which postoperative imaging was performed. ${ }^{24}{ }^{43}$ Cornell and colleagues showed that individuals showing improved global left ventricular function after revascularisation had incomplete recovery at 3-6 months, with late improvement occurring up to a median of 14 months after CABG in approximately one third of these subjects. ${ }^{24}$ Moreover, in the absence of routine postoperative angiography, we were unable to exclude incomplete revascularisation or the occurrence of further native vessel/graft occlusion between the immediate postoperative and second MRI study.

The use of manual quantitative analysis, a factor common to ourselves and Gunning and colleagues, ${ }^{17}$ was time consuming, and prone to both inter- and intraobserver variability. Application of semi-automated edge detection analysis improved the sensitivity and specificity of MRI as a predictor of preoperative myocardial viability, and reduced interobserver variability. The occurrence of respiratory motion artefacts, caused by the lengthy imaging time inherent to the FISP sequence, resulted in comparatively poor images for some subjects. This issue can now be eliminated by using segmented k-space breath hold techniques not available on the scanner used at the outset of this study. ${ }^{44}{ }^{45}$ Sayad and colleagues have suggested that myocardial tagging may offer advantages, though this technique is also a new development and as yet unsubstantiated. ${ }^{46}$

\section{CONCLUSIONS}

In keeping with previous data, we have found dobutamine MRI scanning to be a moderately sensitive, and reasonably specific, predictor of functional recovery after CABG in akinetic or severely hypokinetic myocardium. The use of semi-automated edge detection improved both the sensitivity and specificity of the technique, and may also reduce observer error. Recent developments in rapid breath hold MRI have shown superior results. These are likely to be further improved by the use of multiple dose imaging regimens as scanning times become progressively shorter.

This work was performed with a grant from the British Heart Foundation

1 Mickleborough LL, Maruyama H, Takagi Y, et al. Results of revascularisation in patients with severe left ventricular dysfunction. Circulation 1995;92 (suppl II):II-73-9.

2 Rahimtoola SH. The hibernating myocardium. Am Heart $\mathcal{f}$ 1989;117:211-21.

3 Dilsizian V, Rocco TP, Freedman NMT, et al. Enhanced detection of ischemic but viable myocardium by the reinjection of thallium after stress-redistribution imaging. N Engl F Med 1990;323:141-6.
4 Marzullo P, Parodi O, Reisenhofer B, et al. Value of rest-thallium-201/technetium-99m sestamibi and dobutamine echocardiography for detecting myocardial viability. Am f Cardiol 1993;71:166-72.

5 Arnese M, Cornel JH, Salustri A, et al. Prediction of improvement of regional left ventricular function after surgical revascularization. A comparison of low-dose dobutamine echocardiography with $201 \mathrm{Tl}$ single-photon emission computed tomography. Circulation 1995;91:2748-52.

6 Haqe T, Furukawa MD, Takahashi M, et al. Identification of hibernating myocardium by dobutamine stress echocardiography: comparison with thallium-201 reinjection imaging. Am Heart f 1995; 130:553-63.

7 Vanoverschelde JLJ, D'Hondt AM, Marwick T, et al. Headto-head comparison of exercise-redistribution-reinjection hallium single-photon emission computed tomography thallium single-photon emission computed tomography and low dose dobutamine echocardiography for prediction of reversibility of chronic left ventricular
dysfunction. $7 \mathrm{Am}$ Coll Cardiol 1996;28:432-42.

8 Udelson JE, Coleman PS, Metherall J, et al. Predicting recovery of severe regional ventricular dysfunction: comrecovery of severe regional ventricular dysfunction: com-
parison of resting scintigraphy with ${ }^{201} \mathrm{Tl}$ and ${ }^{99} \mathrm{Tc}$ parison of resting scintigraphy with
sestamibi. Circulation 1994;89:2552-61.

9 Marzullo P, Sambucetti G, Parodi O, et al. Regional concordance and discordance between rest thallium 201 and sestamibi imaging for assessing tissue viability: comparison with postrevascularisation functional recovery. fournal of Nuclear Cardiology 1995;2:309-16.

10 Maublant JC, Citron B, Lipiecki J, et al. Rest technetium $99 \mathrm{~m}$ sestamibi in hibernating myocardium. Am Heart $\mathcal{f}$ 1995;129:306-14.

11 Cigarroa CG, de Fillipe CR, Brickner ME, et al. Dobutamine stress echocardiography identifies hibernating myocardium and predicts recovery of left ventricular function after coronary revascularisation. Circulation 1993;88: 430-6.

12 Perrone-Filardi P, Pace L, Prastaro M, et al. Dobutamine echocardiography predicts improvement of hypoperfused dysfunctional myocardium after revascularisation in patients with coronary artery disease. Circulation 1995;91: 2556-65

13 Afridi I, Kleiman NS, Raizner AE, et al. Dobutamine echocardiography in myocardial hibernation. Optimal dose and accuracy in predicting recovery of ventricular function after coronary angioplasty. Circulation 1995;91:663-70.

14 Gunning MG, Chau TP, Harrington D, et al. Hibernating myocardium: clinical and functional response to revascularisation. Eur $\mathcal{F}$ Cardiothorac Surg 1997;11:1105-12.

15 Castro PF, Bourge RC, Foster RE. Evaluation of hibernating myocardium in patients with ischaemic heart disease. Am 7 Med 1998;104:69-77.

16 Matter C, Nagel E, Stuber M, et al. Assessment of systolic and diastolic LV function by MR myocardial tagging. Basic Res Cardiol 1996;91(suppl 2):23-8.

17 Gunning MG, Anagnostopoulos C, Knight CJ, et al. Comparison of 201TI, 99m Tc-tetrofosmin, and dobutamine magnetic resonance imaging for identifying hibernating myocardium. Circulation 1998;98:1869-74.

18 Baer FM, Smolarz K, Theissen P, et al. Dobutaminegradient echo MRI: a functional and morphologic approach to the detection of residual myocardial viability. Circulation 1995;91:1006-15.

19 Baer FM, Voth E, LaRosee K, et al. Comparison of dobutamine-transoesophageal echocardiography and dobutamine magnetic resonance imaging for the detection of residual myocardial viability. Am f Cardiol 1996;78:425-9.

20 Baer FM, Theissen P, Schneider CA, et al. Dobutamine magnetic resonance imaging predicts contractile recovery of chronically dysfunctional myocardium after successful revascularisation. $\mathcal{7}$ Am Coll Cardiol 1998;31:1040-8.

21 Feigenbaum H. Exercise echocardiography. F Am Soc Echocardiogr 1988;1:161-6.

22 Kaul S. Dobutamine echocardiography for determining myocardial viability after reperfusion: experimental and clinical observations. Eur Heart f 1995;16(suppl M): 17-23.

23 Cornel JH, Bax JJ, Elhendy A, et al. Predictive accuracy of echocardiographic response of mildly dyssynergic myocardial segments to low-dose dobutamine. Am f Cardiol 1997; 80:1481-4.

24 Cornel JH, Bax JJ, Elhendy A, et al. Biphasic response to dobutamine predicts improvement of global left ventricular function after surgical revascularisation in patients with stable coronary artery disease. $7 \mathrm{Am}$ Coll Cardiol 1998;31: 1002-10.

25 Sechtem U, Sommerhoff BA, Markiewicz W, et al. Regional left ventricular wall thickening by magnetic resonance imaging: evaluation in normal persons and patients with global and regional dysfunction. Am f Cardiol 1987;59: $145-51$.

26 Semelka RC, Tomei E, Wagner S, et al. Normal left ventricular dimensions and function: interstudy reproducibility of measurements with cine-MR imaging. Radiology 1990;174:763-8

27 Waiter GD, McKiddie FI, Redpath TW, et al. Determination of normal regional left ventricular function from cine-MR images using a semi-automated edge detection method. Magnetic Resonance Imaging 1999;17:99-107.

28 Bland M. An introduction to medical statistics. Oxford: Oxford University Press, 1987.

29 Alderman EL, Fisher LD, Litwin P, et al. Results of coronary artery surgery in patients with poor left ventricular ary artery surgery in patients with poor left
function (CASS). Circulation 1983;68:785-95.

30 Piggot JD, Kouchoukos NT, Oberman A, et al. Late results of surgical and medical therapy for patients with coronary 
artery disease and depressed left ventricular function. $7 \mathrm{Am}$ Coll Cardiol 1985;5:1036-45.

31 Eitzman D, Al-Alouar ZR Kanter HL, et al. Clinical outcome of patients with advanced coronary artery disease after viability studies with positron emmision tomography. 7 Am Coll Cardiol 1992;20:559-65.

32 DiCarli MF, Davidson M, Little R, et al. Value of metabolic imaging with positron emission tomography for evaluating prognosis in patients with coronary artery disease and left ventricular dysfunction. Am f Cardiol 1994;73:527-33.

33 Bax JJ, Wijns W, Cornell JH, et al. Accuracy of currently available techniques for prediction of functional recovery after revascularisation in patients with left ventricular dysfunction due to chronic coronary artery disease: comparison of pooled data. F Am Coll Cardiol 1997;30:1451-60.

34 Sawada S, Elsner G, Segar DS, et al. Evaluation of patterns of perfusion and metabolism in dobutamine-responsive myocardium. $\mathcal{f} \mathrm{Am}$ Coll Cardiol 1997;29:55-61.

35 Sklenar J, Ismail S, Villaneuva FS, et al. Dobutamine echocardiography for determining the extent of myocardial salvage after reperfusion. An experimental evaluation. Circulavage after reperfusion.
tion $1994 ; 90: 1502-12$.

36 Melendez JC, McCrank E. Anxiety-related reactions wih magnetic resonance imaging examinations. FAMA 1994 271:981-2

37 Pierard LA, de Landsheere CM, Berther C, et al. Identification of viable myocardium by echocardiography during dobutamine infusion in patients with myocardial infarction thrombolytic therapy: comparison with positron emission tomography. $\mathcal{F}$ Am Coll Cardiol 1990;15:1021-31.

38 Eslner G, Sawada S, Foltz J, et al. Dobutamine stimulation detects stunned but not hibernating myocardium [abstract]. Circulation 1994;90(suppl I):I-117.
39 Sambucetti G, Parodi O, Marzullo P, et al. Regional myocardial blood flow in stable angina pectoris associated with isolated significant narrowing of either the left anterior descending or left circumflex artery. Am f Cardiol 1993;72: 990-4

40 Vanoverschelde JLJ, Wijns W, Depre C, et al. Mechanisms of chronic regional postischaemic dysfunction in humans. New insight for the study of noninfarcted collateraldependent myocardium. Circulation 1993;85:1347-53.

41 Bisi G, Sciagra R, Santoro GM, et al. Technetium-99m sestamibi imaging with nitrate infusion to detect viable hibernating myocardium and predict post revascularisation recovery. $\mathcal{F}$ Am Coll Cardiol 1994;24:1282-9.

42 Maurea S, Cuocolo A, Soricelli A, et al. Enhanced detection of viable myocardium by technetium-99m-MIBI imaging after nitrate administration in chronic coronary artery disease. $\mathcal{F}$ Nucl Med 1995;36:1945-52.

43 Vanoeverschelde JL, Melin JA, Depre C, et al. Time-course of functional recovery of hibernating myocardium after of functional recovery of hibernating myocardium after
coronary revascularisation [abstract]. Circulation 1994; 90(suppl I):I-378.

44 Bluemke DA, Boxerman JL, Atalar E, et al. Segmented K-space cine breath-hold cardiovascular MR imaging. 1. Principles and technique. Am f Roentgenol 1997;169:395400.

45 Leung DA, Debatin JF, Wildermuth S, et al. Cardiac imaging: comparison of two-shot echo-planar imaging with fast segmented $\mathrm{K}$-space and conventional gradient-echo cine acquisitions. $\mathcal{F}$ Mag Reson Imaging 1995;5:684-8.

46 Sayad DE, Willet DL, Hundley WG, et al. Dobutamine magnetic resonance imaging with myocardial tagging quantitatively predicts improvement in after revascularization. Am f Cardiol 1998;82:1149-51. 